\title{
Cross sectional profiles of systolic flow velocities in left ventricular outflow tract of normal subjects
}

\author{
Markku Kupari, Pauli Hekali, Veli-Pekka Poutanen
}

\begin{abstract}
Background-The idea that blood passes through the left ventricular outflow tract with a flat velocity profile has recently been questioned.

Objective-To construct flow velocity profiles by magnetic resonance imaging over the whole cross sectional subaortic flow area in normal subjects.

Subjects-Nine people without heart disease aged 25 to 56 .

Design-Cardiac gated cine magnetic resonance images were produced with a 1.0 T magnet perpendicular to the longitudinal axis of the left ventricular outflow tract 0.5 to $1.0 \mathrm{~cm}$ below the aortic annulus in mid-systole. Velocity was encoded every 30-40 ms throughout systole in the direction of flow perpendicular to the image plane.
\end{abstract}

Main outcome measures-Systolic velocity-time curves reconstructed in nine different regions (area of each circle 0.2 $\mathrm{cm}^{2}$ ) of the subaortic flow area. The systolic peak velocity and the mean flow rate were taken as indices of regional flow. Results-The spatial inhomogeneity of the peak velocity, calculated as the percentage ratio of the range of the regional measurements to their mean, averaged $18 \cdot 2 \%(5 \cdot 0 \%)$, and the inhomogeneity of the mean flow rate was $19 \cdot 2 \%(3 \cdot 5 \%)$. There were significant trends $(P<0.05)$ across the regional measurements towards highest peak velocities and mean systolic flow rate in the anteromedial sector of the subaortic flow area. The peak systolic velocity in the centre of the flow area averaged $98(8) \mathrm{cm} / \mathrm{s}$ while the coincident spatial average was 94 (5) $\mathrm{cm}(\mathbf{P}=$ 0.013).

Conclusions-The distribution of systolic velocities across the left ventricular outflow tract is skewed towards fastest flow in the anteromedial sector of the flow area. The peak velocities measured in the centre of the flow area slightly overestimate the coincident spatial average velocities.

(Br Heart f 1995;74:34-39)

Keywords: systolic flow velocity; left ventricular outflow tract; cross sectional profiles

Profiles of intracardiac flow velocity have only recently become available by Doppler ultrasonography, and it has become apparent that the complexity of intracardiac flow has not been fully understood and that certain established concepts may have been mistaken simplifications of flow physiology. ${ }^{1-3}$ The idea of spatial homogeneity of flow in the left ventricular outflow tract, fundamental though it is to the measurement of stroke volume and aortic valve area by Doppler ultrasonography, ${ }^{45}$ has also been raised for critical re-evaluation. The reason is that several studies using colour Doppler ultrasonography, including ours, have exposed faster systolic flow velocities along the septal or anterior margins of the left ventricular outflow tract than laterally or posteriorly. ${ }^{167}$ Owing to the limitations of the Doppler method, however, these studies could not produce spatially complete cross sectional velocity maps but only the range of velocities along one or two diagonals across the flow area.

Velocity encoded cine magnetic resonance imaging enables non-invasive determination of flow velocities across any section of the heart or great vessels. ${ }^{8}$ The method is well validated $^{9-11}$ and has been used to study flow profiles in the aorta ${ }^{12-14}$ and pulmonary artery. ${ }^{15}$ We used this technique to study cross sectional velocity profiles in the left ventricular outflow tract in subjects without heart disease. Specifically, we aimed at assessing whether there are consistent differences in flow through different sectors of the subaortic annulus and whether the recordings taken centrally are representative of the average flow.

\section{Subjects and methods}

SUBJECTS

We studied nine subjects, (five men and four women) aged 25 to 56 (mean age 37 ). One man had well controlled hypertension without any signs that the left ventricle was affected; the others were free of any history, symptoms, or signs of cardiovascular disease. All were members of the hospital staff and gave oral informed consent to this investigation. The study protocol was approved by the local ethics committee.

\section{FLOW VELOCITY MAPPING BY MAGNETIC RESONANCE IMAGING \\ Basic principles}

The principles of flow velocity mapping by gradient echo magnetic resonance imaging have been presented in detail..$^{8916}$ In short, the magnetic resonance signal possesses phase in addition to amplitude, and the velocity of the moving proton spins can be encoded into the 

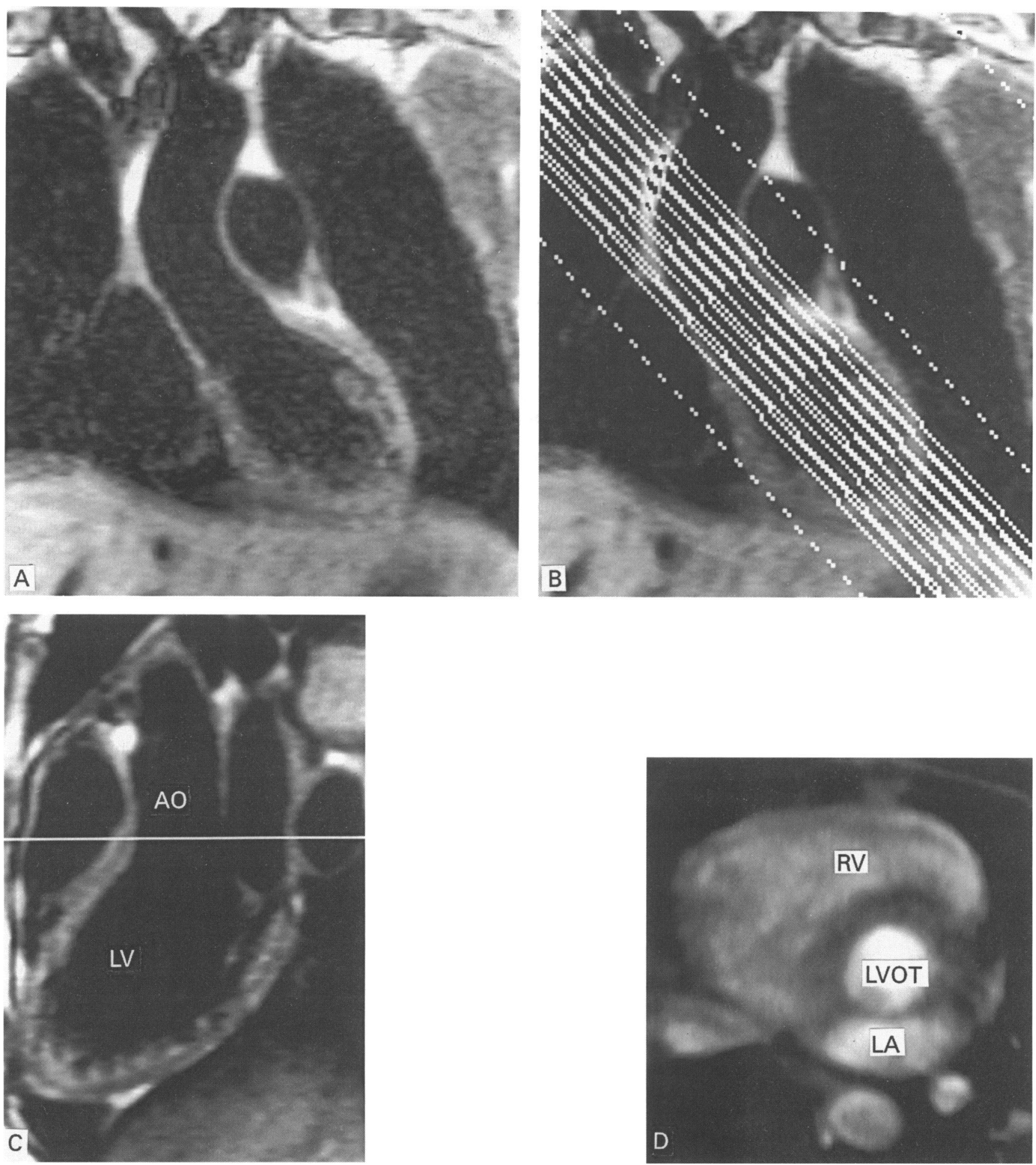

Figure 1 Image plane for the phase contrast study of flow in the left ventricular outflow tract. A: Coronal spin echo image to localise the left ventricular apex and the aortic root. B: The same image with a superimposed set of eight parallel oblique long axis planes for a multislice spin echo study of the outflow tract. C: Mid-systolic long axis spin echo image showing the phase contrast image plane (transverse line) set to transect the outflow tract between the left ventricle and the aorta. D: Systolic frame from a cine study to help visualise the structural relations of the left ventricular outflow tract in the phase contrast image plane. Panels $C$ and $D$ show that the outflow tract borders anteriorly on the ventricular septum and right ventricle and posteriorly on the base of the anterior mitral leaflet and the left atrium. Note that the septal part of the circumference of the flow area extends from about 730 to 130 and thus forms most of the anteromedial wall of the outflow tract (panel $D$ ). $L A$, left atrium; $L V$, left ventricle; $L V O T$, left ventricular outflow tract; $R V$, right ventricle.

signal phase. Phase images then become velocity maps showing the velocity of the spins in each individual voxel of the image. To remove phase changes due to inhomogeneity in the magnetic field or the effects of chemical shift in the subject, two images with different encoding gradients are acquired, interleaved, and subtracted to give the velocity map. This procedure makes a sepa- rate velocity calibration unnecessary. ${ }^{9}$ Velocity and higher orders of motion can be encoded either through the image plane or within the plane.

Flow velocity mapping

We used a superconductive $1.0 \mathrm{~T}$ imager (Siemens Magnetom 42, Erlangen, Germany), a body coil, and electrocardiographic triggering. 
The subjects were positioned in the scanner in a comfortable supine position and asked to lie as still as possible during the imaging, which took about 30 minutes. Heart rate and rhythm were monitored throughout the study and remained normal in all subjects; the man with hypertension had moderate drug related sinus bradycardia.

The imaging started with acquisition of a $\mathrm{T} 1$ weighted multislice series of coronal spin echo images to localise the aortic root and the left ventricular apex (figure 1(A)). Another multislice series of oblique $\mathrm{T} 1$ weighted spin echo studies was obtained thereafter to image the left ventricular outflow tract during systole, from 100 to $300 \mathrm{~ms}$ after the $R$ wave peak (figure 1(B) and (C)). A mid-systolic image of the outflow tract was selected, and the image plane for the phase contrast study was positioned $0.5-1 \mathrm{~cm}$ below the level of the aortic annulus (figure $1(\mathrm{C})$ and (D)). Velocity encoding was made in the direction perpendicular to the image plane-that is, parallel to the longitudinal axis of the outflow tract. The field of view was $350 \times 350 \mathrm{~mm}$ and the matrix size was $192 \times 256$, resulting in pixel dimensions of $1.8 \times 1.4 \mathrm{~mm}$; the slice thickness was $6 \mathrm{~mm}$. The echo time was 6 $\mathrm{ms}$ and the repetition time $30-40 \mathrm{~ms}$. Since the echo time was short and the velocities of interest were in the physiological range, phase shifts due to acceleration and deceleration were neglected. ${ }^{10}$ The upper limit of the velocity scale was set at $150 \mathrm{~cm} / \mathrm{s}$. The study was made using two interleaved sequences with identical echo time but with different velocity

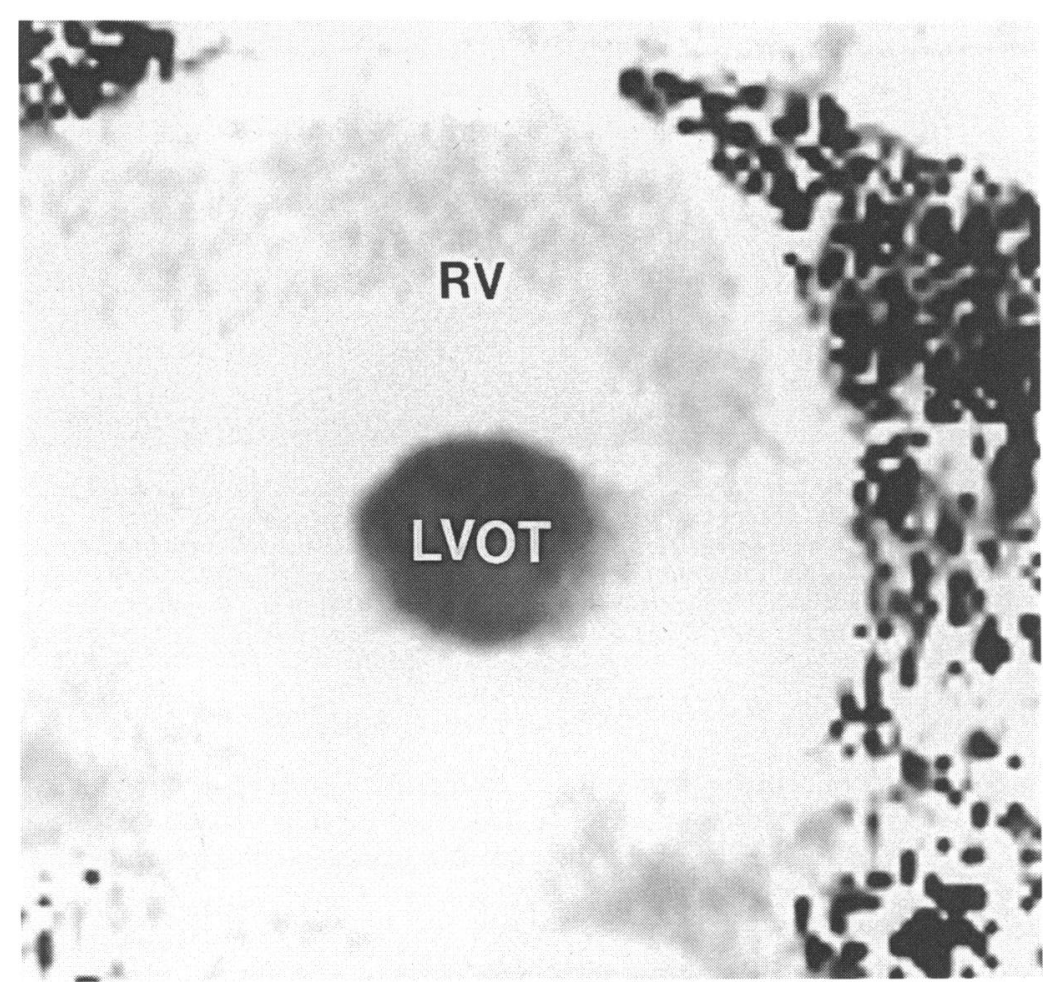

Figure 2 Systolic phase contrast image showing the flow area of the left ventricular outflow tract. The perspective of the image is identical with that of figure 1 (D). LVOT, left ventricular outflow tract; $R V$, right ventricle. encoding gradients in the same direction. For each phase of the cardiac cycle the interleaved images were subtracted pixel by pixel after Fourier transformation to reconstruct magnitude and phase images (figure 2). The final result was a series of flow velocity maps every $30-40 \mathrm{~ms}$ during left ventricular ejection. The velocity profiles could also be reconstructed as a series of three dimensional surface maps illustrating the distribution of the instantaneous velocities across the subaortic annulus. These were viewed as a slow motion video to help assess the dynamics of flow both in space and in time. Selected frames were documented as three dimensional wire maps.

\section{Velocity analyses}

To measure the differences in spatial flow velocity, systolic velocity time curves were reconstructed in nine different areas of the subaortic cross section of the left ventricular outflow tract. Each region of interest encompassed an area of $0.2 \mathrm{~cm}^{2}$. The measurement regions were positioned manually over the phase contrast image, one in the centre of the outflow tract and the rest radially and more peripherally in eight sectors of $45^{\circ}$ of the flow area. Owing to the contractions of the left ventricle the location of the outflow area in the image plane changed cyclically; the mean amplitude of the shift of the centre was 3.2 $\mathrm{mm}$ in the mediolateral direction and $3.9 \mathrm{~mm}$ in the anteroposterior direction. Therefore to keep the relative positions of the measurement areas unaltered, the regions of interest were defined frame by frame. The regional instantaneous velocities were calculated as averages over the pixels included in each measurement area. To reconstruct regional velocity-time curves, the velocity at each phase was plotted against the delay of the phase from the $R$ wave. The regional systolic peak velocity and mean systolic volumetric flow rate were determined; mean systolic volumetric flow rate was calculated as the product of the temporal mean systolic velocity and the known size of the flow area. Spatial average velocities were analysed similarly but using regions of interest encircling the entire subaortic annulus. All velocity data were corrected using a circular background region with an area of $2.4 \mathrm{~cm}^{2}$ in the periphery of the liver. The velocity in this area with no flow was subtracted from all velocities found in the outflow tract. Typically, the background velocities were in the range of $-2 \mathrm{~cm} / \mathrm{s}$ to $2 \mathrm{~cm} / \mathrm{s}$.

To evaluate the reproducibility of the velocity measurements, two blinded determinations of the regional and spatial average peak systolic velocities in the left ventricular outflow tract were made in all nine subjects studied. The reproducibility was calculated for each subject and for each measurement region as the percentage ratio of the absolute difference between the paired data to their mean. The average reproducibility percentage was $<5 \%$ in seven of the nine interrogated flow areas and $<10 \%$ in all areas. The reproducibility of the spatial average peak velocity was $3 \cdot 4 \%(3 \cdot 7 \%)$. 

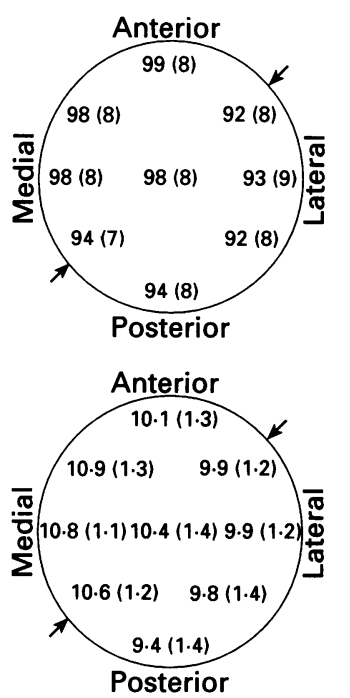

Figure 3 Mean (SD) regional peak systolic velocities in $\mathrm{cm} / \mathrm{s}$ (top) and mean systolic flow rates in $\mathrm{ml} / \mathrm{s}$ (bottom) in the different measurement areas across the left ventricular outflow tract. The flow area is depicted schematically as a circle with the same orientation as that of figure 1 (D); the anteromedial circumference between the arrows margin of the outflow tract. Note the location of fastest flow in the anteromedial sector. corresponds to the septal

\section{STATISTICAL ANALYSIS}

Repeated measurements analysis of variance was used to assess whether there were significant overall differences in velocity recorded in the different regions of the subaortic annulus. Selected pairwise comparisons were made with Student's paired $t$ test. Bivariate correlation coefficients were calculated by Pearson's product-moment method. The data are given as means (SD). Two tailed $P$ values $<0.05$ were considered to be significant. All calculations and the graphical reconstruction of the three dimensional velocity profiles were performed on a microcomputer using commercially available software. ${ }^{17}$

\section{Results}

The spatial variation of the peak systolic velocity across the subaortic annular flow area, calculated for each individual as the percentage ratio of the width of the range of the nine regional measurements to their mean averaged $18 \cdot 2 \% \quad(5 \cdot 0 \%)$ (range $12 \cdot 2 \%$ $28.5 \%)$. The corresponding spatial inhomogeneity of the mean systolic flow rate was $19.2 \%$ (3.5\%) (range $14.8 \%-24.6 \%$ ). Compared with the random variation of determining the regional velocities (see methods section), these figures are clearly higher, suggesting true spatial flow inhomogeneity.

The regionally measured peak systolic velocities and mean systolic flow rates are summarised in figure 3 for the whole study group. The data show that the highest systolic velocities and flow rates were located anteromedially in the subaortic annular flow area. The direction of the skewness of the flow pro-

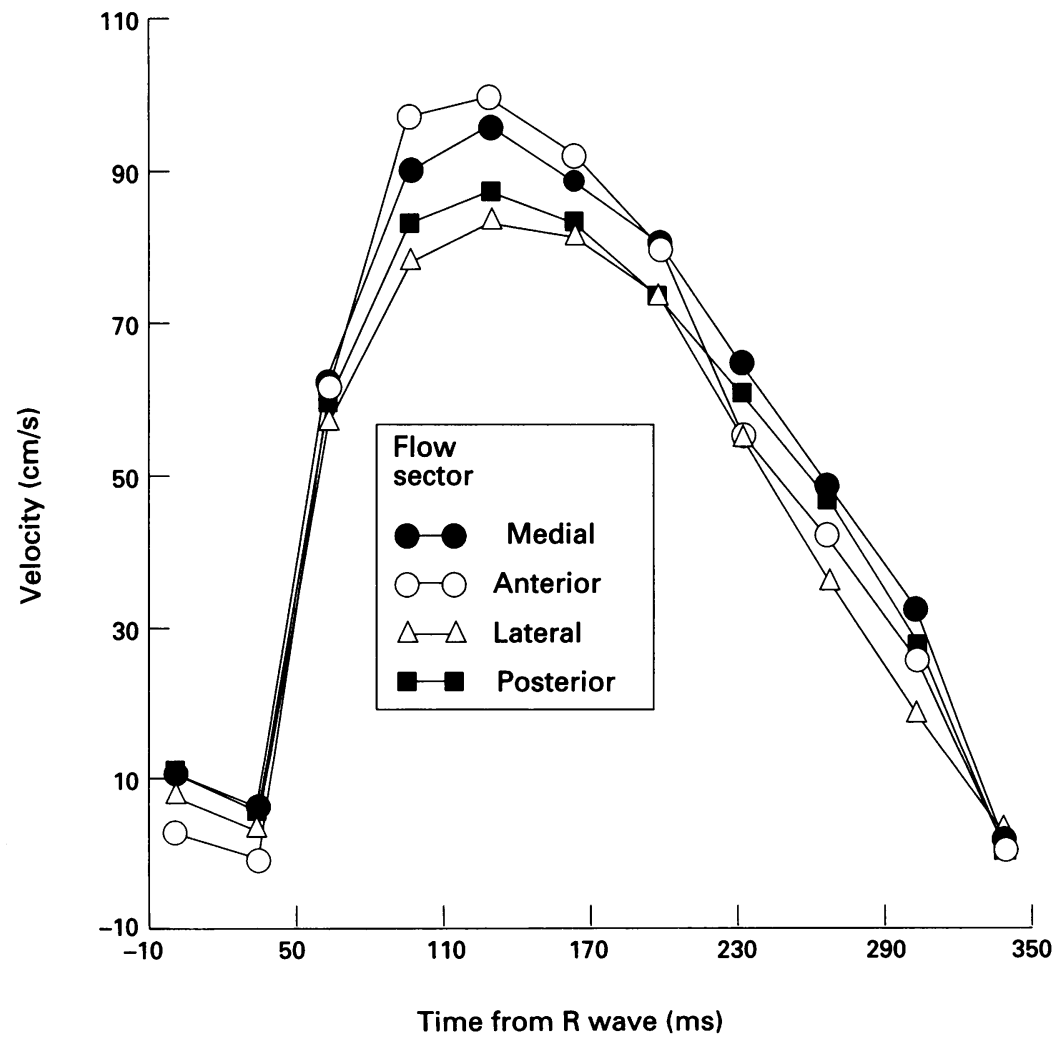

Figure 4 Regional systolic velocity-time curves in the medial, anterior, lateral, and posterior sectors of the left ventricular outflow tract in one woman aged 31. file was consistent from subject to subject as shown by significant overall trends by repeated measurements analysis of variance across both regional peak systolic velocities ( $F$ $=2.8, P=0.011)$ and mean flow rates $(F=$ $5.0, P<0.001)$. Figure 4 shows selected regional systolic velocity-time curves in one subject, and figure 5 shows a series of three dimensionally reconstructed systolic velocity profiles across the left ventricular outflow tract in another. Both figures show the trend towards highest flow velocities anteromedially in the subaortic annular flow area. Overall, the spatial maximum of the peak systolic velocity was located in the anteromedial flow area in eight of the nine subjects. Temporally, the regional velocity differences were most prominent around the mid-systolic flow peak.

The total mid-systolic flow area of the subannular left ventricular outflow tract averaged $3.4(0.4) \mathrm{cm}^{2}$ in the study group. The spatial average peak systolic velocity, representing the mean of peak velocities in all voxels included in the flow area, was $94(5) \mathrm{cm} / \mathrm{s}$; the corresponding mean systolic flow rate was $172(30) \mathrm{ml} / \mathrm{s}$. When the measurements made in the centre of the flow area (see figure 3) were compared with the spatial average data, the central peak velocity was on average $4.0 \%$ larger than the spatial mean value (range $-1.0 \%$ to $10 \cdot 8 \% ; P=0.019$ ).

\section{Discussion}

To our knowledge, this is the first reconstruction of spatially complete cross sectional flow velocity maps in the left ventricular outflow tract in human subjects. The velocity profiles were non-uniform and showed a consistent trend towards fastest flow through the anteromedial sector of the flow area. The non-uniformity was most conspicuous at the time of peak systolic flow. These results support and complement earlier Doppler studies showing higher velocities septally or anteriorly than laterally or posteriorly in the left ventricular outflow tract. ${ }^{167}$ The data also agree with the reported location of the supravalvar velocity maximum close to the right anterior aortic wall in the aortic root. ${ }^{18}$ Taken all together, the concept of blood traversing the left ventricular outflow tract with a spatially uniform velocity can be considered disproved.

The validity and usefulness of velocity measurements by phase contrast magnetic resonance imaging have been amply documented previously, both with flow phantoms and in intact humans. ${ }^{91116}$ The method has been used to characterise flow profiles in the great arteries $^{13-15}$ but not systematically inside the heart. The determination of flow velocity at each voxel of a vascular or cardiac image is its unique advantage and enables the reconstruction of spatially complete cross sectional flow velocity maps. The image section can be selected freely, and the velocities can be measured, if necessary, in three directions. ${ }^{19}$ However, we measured only flow perpendicular to the image section-that is, parallel to the longitudinal axis of the outflow tract. 

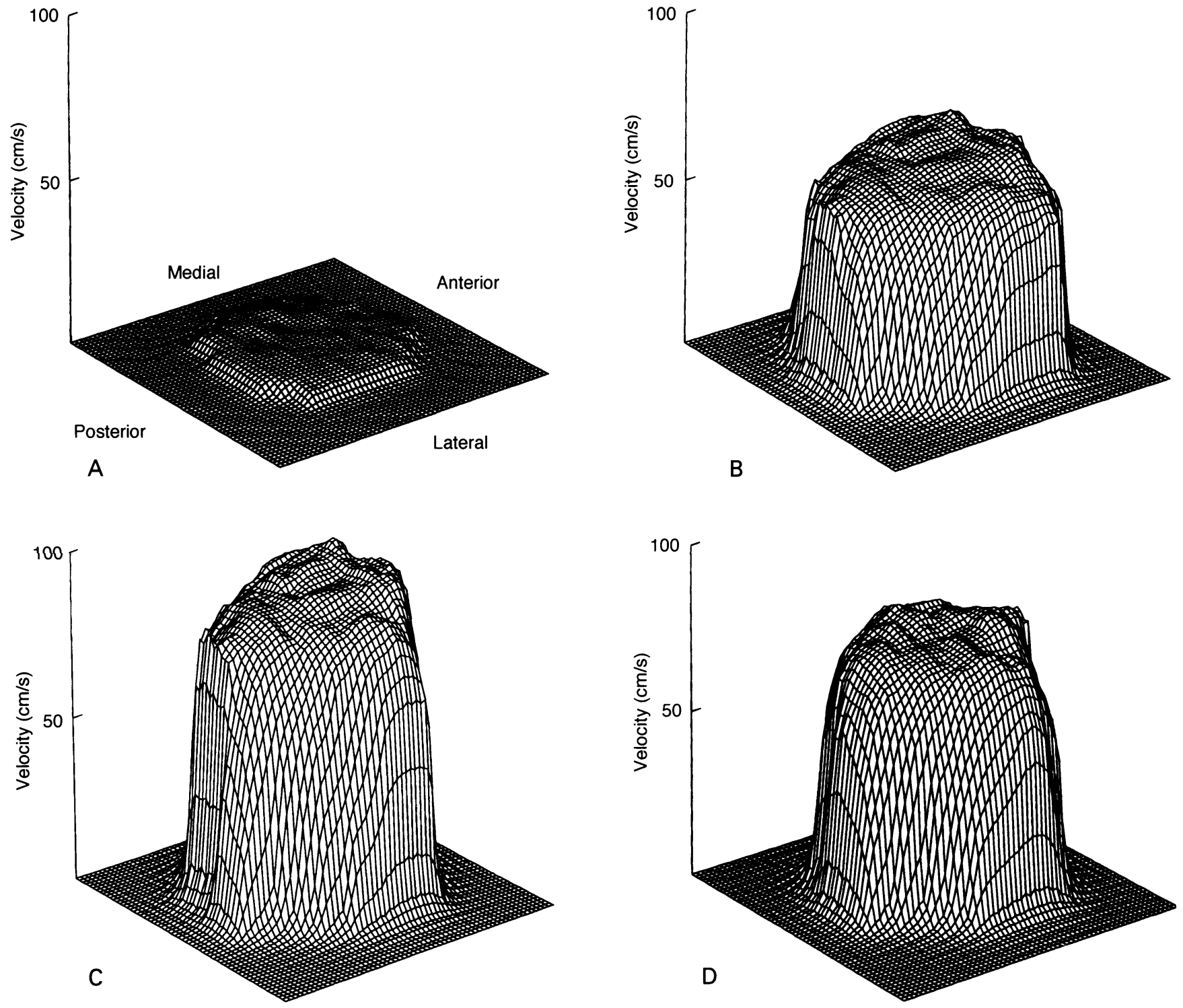

Figure 5 Serial three dimensional flow velocity profiles across the left ventricular outflow tract in one subject. The orientation of the view is shown in $A$. $A$ and $B$ represent the early phase of ejection, $C$ mid-systole at the time of peak flow, and $D$ and $E$ the late phase of ejection. Note the skewness of the spatial velocities towards fastest flow in the anteromedial flow area, particularly in $B, C$, and $D$.

Although this is a limitation of our data, we emphasise that any flow components in the short axis plane of the left ventricular outflow tract are likely to be negligible and that simultaneous three dimensional velocity encoding would have compromised the temporal resolution of our flow measurements. Owing to the cyclic movements of the heart, as opposed to the fixed image plane, the intracardiac velocity maps do not represent flow profiles in only one anatomical section of the heart. Rather, they show the distribution of velocities in a series of planes that overlap but are not fully identical. In our study the image

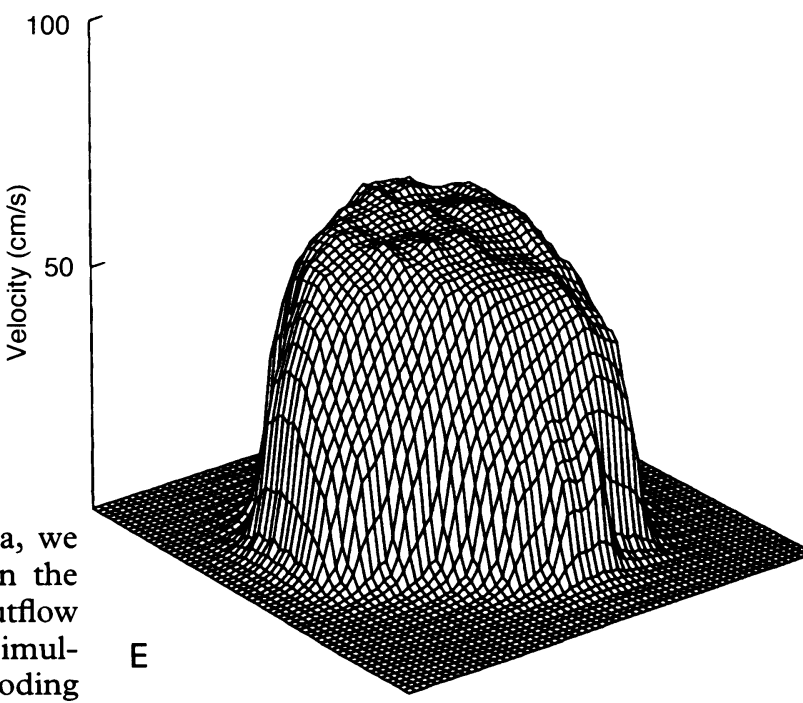

plane was positioned so that the mid-systolic and late systolic flow profiles represented the cross sectional velocity distribution within $0 \cdot 5-1 \mathrm{~cm}$ of the aortic annulus while the early systolic profiles were recorded somewhat more proximally in the outflow tract. This 
problem, however, is not unique to magnetic resonance imaging: the Doppler recordings of subaortic annular velocities suffer from the same limitation of the aortic annulus moving in relation to a spatially fixed sampling gate.

Although our results agree in principle with the earlier observations with colour Doppler ultrasonography, ${ }^{167}$ spatial velocity differences were not as large as suggested by some of the Doppler studies. ${ }^{6}$ We emphasise, however, that the velocity profiles generated by Doppler ultrasonography are not fully comparable with those reconstructed by magnetic resonance imaging because Doppler reflects the distribution of velocities along only one diagonal across the left ventricular outflow tract whereas the magnetic resonance imaging covers the whole cross sectional flow area.

Velocity recordings made in the centre of the subaortic annular flow area have been reported to represent the spatial mean flow, notwithstanding the skewness of the flow profiles. ${ }^{6}$ In the study of Zhou et al, the velocities measured at the midpoint of the aortic annular diameter were not significantly different from the average velocities along the same diameter, although a trend toward overestimation by $6 \%$ to $7 \%$ was observed. ${ }^{6}$ We found that the difference between the central and spatial average velocities was even smaller (4\%), though significant. Together these data suggest that velocity measurements in the centre of the flow area are close to, but may slightly overestimate, the spatial average velocities. We emphasise, however, that we studied a small group of people without heart disease and our observations represent normal flow in the left ventricular outflow tract. Whether the flow profiles in patients with valvar or myocardial heart disease are skewed in the same way and to the same extent is unknown. According to some preliminary Doppler data, the non-uniformity of subaortic flow is considerable even in aortic valve stenosis and can result in a wide range of aortic valve areas calculated by the continuity equation. ${ }^{20}$

We could not study mechanisms of the skewed subaortic annular velocity profiles. One contributing factor could be the difference of the longitudinal axis of the left ventricular outflow tract from the axis of the body of the left ventricle. ${ }^{167}$ This misalignment results in angular deviation of flow, which could distort the velocity profile simply because flow in a curved tube is faster along the inner curvature during the first part of the bend. ${ }^{21}$

The practical message of our work is that owing to the skewness of the instantaneous flow profiles the velocities recorded in a small area of the left ventricular outflow tract may not represent the spatial average flow. Nevertheless, assessing the systolic velocities centrally in the subaortic annular flow area may provide results that at least in normal circumstances are fairly close to the coincident spatial average velocities. Finally, our work shows the usefulness of phase contrast magnetic resonance imaging in studying the dynamics of intracardiac flow. With improving temporal resolution and simultaneous three dimensional velocity encoding, this technique will provide an even more powerful tool for both physiological and clinical research.

1 Rossvoll O, Samstad S, Torp HG. The velocity distribution in the aortic annulus in normal subjects: a quantitative analysis of two-dimensional Doppler maps. $¥ \mathrm{Am}$ tive analysis of two-dimensional
Soc Echocardiogr 1991;4:367-78.

2 Samstad SO, Torp HG, Linker DT, et al. Cross sectional early mitral flow velocity profiles from colour Doppler. Br Heart ₹ 1989;62:177-84.

3 Samstad SO, Rossvoll O, Torp HG, Skjaerpe T, Hatle L. Cross-sectional early mitral flow-velocity profiles from colour Doppler in patients with mitral valve disease. Circulation 1992;86:748-55.

4 Lewis JF, Kuo LK, Nelson JG, Limacher MC, Quinones MA. Pulsed Doppler echocardiographic determination of stroke volume and cardiac output: clinical validation of stroke volume and cardiac output: clinical validation of two new method

5 Skjaerpe T, Hegrenaes L, Hatle L. Noninvasive estimation of valve area in patients with aortic stenosis by Doppler ultrasound and two-dimensional echocardiography. Circulation 1985;72:810-8

6 Zhou YG, Faerestrand S, Matre K, Birkeland S. Velocity distributions in the left ventricular outflow tract and the aortic anulus measured with Doppler colour flow mapaortic anulus measured with Doppler colour flow map-

7 Kupari M, Koskinen P. Systolic flow velocity profile in the left ventricular outflow tract in persons free of heart the left ventricular outflow tract in pers
disease. Am $₹$ Cardiol 1993;72:1172-8.

8 Mohiaddin RH, Longmore DB. Functional aspects of cardiovascular nuclear magnetic resonance imaging: techniques and applications. Circulation 1993;88:264-81

9 Walker MF, Souza SP, Dumoulin CL. Quantitative flow measurement in phase contrast $M R$ angiography. f Comput Assist Tomogr 1988;12:304-13.

10 Maier D, Meier S, Boesiger P. Quantitative flow measurements on phantoms and on blood vessels with $M R$ Magn Reson Med 1988;8:25-34.

11 Firmin DN, Nayler LG, Klipstein RH, Underwood SR, Rees RSO, Longmore DB. In vivo validation of MR velocity imaging. F Comput Assist Tomogr 1987;11:751-6.

12 Sondergaard L, Lindvig K, Hildebrandt $\mathrm{P}$, et al. Quantification of aortic regurgitation by magnetic resonance velocity mapping. Am Heart $\mathcal{f}$ 1993;125:1081-90.

13 Klipstein RH, Firmin DN, Underwood SR, Rees RSO, Longmore DB. Blood flow pattern in the human aorta studied by magnetic resonance. $B r$ Heart $\mathcal{F} 1987 ; 58$ : 316-23.

14 Meier SE, Maier D, Boesiger P, Moser UT, Vieli A. Human abdominal aorta: comparative measurements of blood flow with MR imaging and multigated Doppler US. Radiology 1989;171:487-92.

15 Kondo C, Caputo GR, Masui T, et al. Pulmonary hypertension: pulmonary flow quantification and flow profile analysis with velocity-encoded cine $M R$ imaging. Radiology 1992;183:751-8.

16 Nayler GL, Firmin DN, Longmore DB. Blood flow imaging by cine magnetic resonance. $\mathcal{f}$ Comput Assist Tomogr 1986;10:715-22.

17 Systat. SYSTAT - SYSGRAPH, version S.D. Evanston, IL: Systat, 1990.

18 Mathison M, Furuse A, Asano K. Doppler analysis of flow velocity profile at the aortic root. $\mathcal{F} \mathrm{Am}$ Coll Cardiol 1988;12:947-54.

19 Kilner P, Wann LS, Firmin D, Longmore D. Three directional magnetic resonance flow imaging of the directional magnetic resonance flow imaging of the human

20 Zhou Y-Q, Faerestrand S, Matre K. The velocity distribution in the left ventricular outflow tract: effect on aortic stenosis area measurement. Circulation 1993;88(suppl): I-450.

21 Hatle L, Angelsen B. Physics of blood flow. In: Hatle L, Angelsen B, eds. Doppler ultrasound in cardiology. Philadelphia: Lea and Febiger; 1985:8-31. 